\title{
DETERMINAÇÃO DE CARBOFURANO EM CALDO DE CANA UTILIZANDO A EXTRAÇÃO LÍQUIDO-LÍQUIDO COM PARTIÇÃO À BAIXA TEMPERATURA E ANÁLISE POR CROMATOGRAFIA LÍQUIDA
}

\author{
Charles Ivo de Oliveira Júnior ${ }^{1}$, Rogério Pacheco Rodrigues ${ }^{1}$, Yuri Alves Oliveira ${ }^{1}$, Adilson \\ Correia Goulart $^{1}$, João Paulo Victorino Santos ${ }^{1}$, Simone Machado Goulart ${ }^{1 *}$ \\ ${ }^{1}$ Instituto Federal de Goiás, Itumbiara, 75524-245, Goiás, Brasil
}

Autor para correspondência: Simone Machado Goulart; e-mail: simone.goulart@ifg.edu.br

Recebido: 3006/2020, Aceito: 11/12/2020

\begin{abstract}
Resumo
O uso de agrotóxicos tem se intensificado para atender à demanda do mercado. O Brasil é o maior produtor de cana-de-açúcar do mundo e uma das suas formas de consumo é o caldo de cana in natura conhecido popularmente como garapa. Não há uma legislação no país que aborde parâmetro para determinação de agrotóxicos em caldo de cana sendo assim a comercialização da bebida não segue nenhum controle de qualidade. $O$ objetivo dessa pesquisa foi avaliar o emprego do método Extração Líquido-Líquido com Partição à Baixa Temperatura (ELLPBT) em relação à seletividade, aplicabilidade e efeito da adição de cloreto de sódio para determinação do agrotóxico carbofurano em caldo de cana e posterior análise de amostras reais de caldo de cana comercializados na cidade de Itumbiara - GO. Este método é simples e eficaz, consumindo pequenas quantidades de solventes e apresenta vantagens em relação às demais técnicas de extração, como praticidade e baixo consumo de amostra e solvente orgânico. A porcentagem de recuperação do carbofurano pelo método ELL-PBT foi de 96,94-102,68\% com coeficientes de variação inferiores à $2,0 \%$. A adição de cloreto de sódio não apresentou mudança estatisticamente significativa na porcentagem de extração do carbofurano e as amostras reais do caldo de cana adquiridas no comércio de Itumbiara - GO, submetidas ao método otimizado, não apresentaram resíduos de carbofurano.
\end{abstract}

Palavras-chave: agrotóxico, cana-de-açúcar, cromatografia

\section{DETERMINATION OF CARBOFURAN IN SUGARCANE JUICE USING LIQUID-LIQUID EXTRACTION WITH LOW TEMPERATURE PARTITIONING AND ANALYSIS BY LIQUID CHROMATOGRAPHY}

\begin{abstract}
The use of pesticides has been intensified to meet market demand. Brazil is the largest sugar cane producers in the world and one of its forms of consumption is raw cane juice popularly known as garapa. There is no legislation in the country that addresses parameters for the determination of pesticides in the sugarcane juice, therefore the marketing of the drink does not follow any quality control. The objective of this research was to evaluate the use of the Liquid-Liquid Extraction with Low Temperature Partitioning (LLE-LTP) method in relation to the selectivity, applicability, and effect of the addition of sodium chloride to determine the pesticide carbofuran in sugarcane juice and subsequent analysis of real samples of sugarcane juice sold in the city of Itumbiara - GO. This method is simple and effective, consuming small amounts of solvents and has advantages over other extraction techniques, such as practicality and low consumption of sample and organic solvent. The percentage of carbofuran extraction by (LLE-LTP) was 96.94-102.68\% with coefficients of variation below 2.0\%. The addition of sodium chloride did not show a statistically significant change in the percentage of carbofuran extraction. Samples of the sugarcane juice acquired in the Itumbiara - GO, submitted to the optimized method did not present any carbofuran residues.
\end{abstract}

Keywords: pesticide, sugarcane juice, chomatography. 


\section{Introdução}

O Brasil é o maior produtor de cana-de-açúcar no mundo. A produção estimada de plantações de cana-deaçúcar para a safra de 2019/20, foi de aproximadamente 615,98 milhões de toneladas. A partir do processamento da cana-de-açúcar são produzidos dois produtos essenciais para a economia do país, o açúcar e o etanol (CONAB, 2020). O setor sucroalcooleiro da região de Itumbiara - GO vem crescendo de forma correspondente ao país, o município localiza-se no interior do estado de Goiás, na divisa com o estado de Minas Gerais. Para manter a demanda do mercado, o setor recorre ao uso intensivo de insumos químicos, como fertilizantes e agrotóxicos (SILVA, et al., 2017; VIEIRA, et al., 2017).

Uma das formas mais populares de utilização da cana-de-açúcar é em forma de caldo, onde a planta é esmagada e o sumo é consumido in natura. $\mathrm{O}$ caldo de cana é conhecido popularmente como garapa, devido ao seu sabor refrescante e propriedades energéticas, o produto se destaca em feiras livres e mercados populares. Para a produção da garapa a cana-de-açúcar utilizada, é proveniente de pequenos produtores. As principais características da garapa são a sua viscosidade e a coloração escura. Basicamente, é composta de água e açúcares, tais como: sacarose; frutose; e glucose (PRATI; CAMARGO, 2008).

Para garantir a qualidade dos alimentos em geral é importante o monitoramento de produtos químicos como os agrotóxicos. Esse monitoramento no Brasil é realizado pela Agência Nacional de Vigilância Sanitária (ANVISA) por meio do Programa de Análise de Resíduos de Agrotóxicos em Alimentos (PARA). Entretanto, não há uma legislação no país que aborde parâmetros para a determinação de agrotóxicos em caldo de cana. Sendo assim, a comercialização da bebida não segue nenhum controle de qualidade, e o desenvolvimento dessa pesquisa é importante para evidenciar uma técnica para determinação de agrotóxicos em caldo de cana, visto que, diversos agricultores fazem uso abusivo de agrotóxicos para potencializar a produção, visando uma maior lucratividade (SILVA, et al., 2017; SOARES; BERNARDI; BORGES, 2017).

A quantidade de agrotóxicos registrados, no Brasil em 2019, foi enorme, sendo 474 novos produtos disponíveis para comercialização e utilização na agricultura, grande parte desses agrotóxicos já são proibidos em países desenvolvidos, devido à sua alta toxicidade e perigos que representam para a saúde pública, animais e meio ambiente (BRASIL, 2020).

Uma pesquisa realizada por Rodrigues et al. (2016) na cidade de Itumbiara identificou que $100 \%$ dos agricultores da região fazem o uso de algum tipo de agrotóxico, os trabalhadores do campo relataram sentir um ou mais sintomas de intoxicação, tais como: dores de cabeça; mal-estar; diarreia; e reações cutâneas. Em se tratando de problemas ambientais, foram relatados a morte de animais, o descarte incorreto de embalagens e o desrespeito aos períodos de carência estabelecido pelos órgãos de fiscalização. Fraga et al. (2016) realizaram um estudo sobre os principais agrotóxicos comercializados ilegalmente no Brasil entre os anos de 2008 e 2009 como resultados os pesquisadores evidenciaram como o contrabando desses produtos podem ser prejudiciais para a saúde do homem, visto que a utilização dos agrotóxicos pode ser maior do que o permitido pelos órgãos de fiscalização, além da aplicação de produtos proibidos para determinadas culturas.

Como mencionado acima, outro problema frequente no país, é o comércio ilegal desses produtos químicos, o agrotóxico carbofurano (metilcarbamato de benzofuranila), por exemplo, foi proibido no Brasil pela RDC $n^{\circ} 185$ em 18 de outubro de 2017. Entretanto, diversos agricultores ainda fazem o uso informal desse composto, uma vez que, o princípio ativo é eficiente no controle de pragas como insetos, nematóides, dentre outras. O carbofurano pertence à classe química dos carbamatos é classificado como extremamente tóxico (classe I). Na cana-de-açúcar, a Ingestão Diária Aceitável (IDA) de resíduos de carbofurano é de $0,00015 \mathrm{mg} \mathrm{kg}^{-1}$ de peso corpóreo/dia (BRASIL, 2017). A Figura 1 exibe a estrutura química do carbofurano.

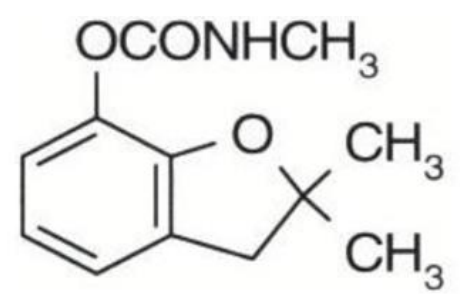

Figura 1 - Estrutura Química do Carbofurano

Quando ingerido, o agrotóxico carbofurano inibe a enzima acetilcolinesterase que degrada a acetilcolina. Esse efeito inibitório facilita o acúmulo de acetilcolina nas sinapses e resulta em movimento descontrolado, paralisia, convulsões ou até morte. Os inseticidas carbâmicos podem ser absorvidos pela pele e pelo trato gastrointestinal. Uma vez absorvidos, são imediatamente distribuídos nos tecidos internos, onde pode causar estresse oxidativo (PULGARIN; BERMEJO; DURÁN, 2018).

A maioria das pesquisas realizadas com o caldo de cana estão voltadas apenas para análises microbiológicas que também são muito importantes para a qualidade da bebida e para saúde do consumidor. Galvão et al. (2019) realizaram uma pesquisa em 
Campo Mourão - PR para avaliar a contaminação microbiológica desta bebida comercializada por vendedores ambulantes na cidade. Os autores constataram índices aceitáveis pela legislação em vigor (102 NMP/mL) identificando contaminação na maioria das amostras analisadas. Já a pesquisa de Mota et al. (2020), foi analisado parasitas que possam ser nocivos à saúde, ocasionando o aparecimento de doenças em amostras de caldo-de-cana comercializados no Distrito Federal. Com base nos resultados, em todas as amostras foi constatado a presença de algum tipo de contaminação, sendo registrado leveduras em $100 \%$ das amostras em $50 \%$ se obteve a presença de Entamoeba sp. em 10\% Taenia sp., em 10\% Giardia sp. e em 10\% Ascaris sp. Em virtude desse fato, verifica-se a importância do monitoramento do caldo de cana tanto por análises microbiológicas como por análises de agrotóxicos, uma vez que, não há medidas de controle de qualidade dessa bebida, o que pôde ser verificado no quesito microbiológico citado anteriormente.

Várias técnicas estão disponíveis para a determinação de resíduos de agrotóxicos em bebidas, entretanto, a maioria dos métodos gera resíduos químicos e impactos ambientais. Atualmente, a técnica utilizada pela ANVISA é o método multirresíduo QuEChERS (Lozowicka, et al., 2016). O método original foi desenvolvido por Anastassiades et al. (2003) para determinar agrotóxicos em frutas e verduras. Como resultado, os pesquisadores apresentaram uma porcentagem de recuperação de agrotóxicos acima de $95 \%$ de recuperação das amostras. Lehotay Maštovská e Lightfield (2005), modificaram o procedimento com a adição $1 \%$ de ácido acético para evitar a variação do $\mathrm{pH}$ e aumentar a porcentagem de recuperação dos agrotóxicos sensíveis a ácidos e bases. Essa técnica tem sido muito utilizada para determinar agrotóxico em nozes pecan, café, caldo de cana, maçã, laranja e uva, por exemplo, nas pesquisas de Barci et al. (2020); Anholeti, Osório e Vieira (2019); Marcílio, Furlani e Tfouni (2019); Furlani et al. (2019); Alcântara et al. (2019).

Tratando-se de estudos para a extração de resíduos de pesticidas em caldo de cana, inúmeros procedimentos foram desenvolvidos, empregando diferentes técnicas de extração, como a Microextração por Absorvente Compacto e QuEChERS (FUMES, et al., 2016; SUGANTHI; BHUVANESWARI; RAMYA, 2018; SAMPAIO et al., 2013).

Sabendo que o carbofurano é aplicado na cultura de cana-de-açúcar, estudos direcionados para a agricultura e o manejo da matéria-prima foram desenvolvidos para a determinação de carbofurano em cana-de-açúcar e solo proveniente do cultivo de cana-de-açúcar (KABIR et al., 2007; SILVA, et al., 2017; GOULART et al., 2018; RAMASUBRAMANIAN; PARAMASIVAM,
2018). Também foi desenvolvido trabalhos como de Raza et al. (2019) que tiveram como objetivo realizar uma pesquisa com produtores de cana-de-açúcar em especial os que utilizavam agrotóxicos. Por meio de entrevistas, foi identificado que grande parte desses agricultores apresentam sintomas de intoxicação e não possui conhecimento técnico para aplicação de agrotóxicos nas culturas de cana-de-açúcar.

A Extração Líquido-Líquido com Partição à Baixa Temperatura (ELL-PBT) é um método simples e eficiente e mostrou-se eficaz na extração de agrotóxicos em bebidas da cor escura. Essa técnica se baseia na partição da amostra em duas fases, sendo uma aquosa e a outra orgânica, com posterior congelamento do sistema, ela tem sido muito utilizada na determinação de carbamatos e outros agrotóxicos em matrizes ambientais e alimentícias. Como já foi mencionado anteriormente, esse método é muito eficaz, utiliza pouca quantidade de solvente e tem boa porcentagem de recuperação (LI; YUAN, 2008). Esse método já foi aplicado em várias matrizes como água, geleia real de abelha, mel, tomate, óleos comestíveis e suco de uva (RODRIGUES et al., 2018; GIROUD, et al., 2019; SILVA, 2020; RODRIGUES et al., 2019; ZHAO et al., 2019).

Alguns autores já verificaram que cloreto de sódio pode influenciar a recuperação dos carbamatos durante o processo de extração, mostrando ser um fator interessante a ser estudado (GOULART et al., 2012).

Alguns estudos preliminares já foram realizados pelos autores desse artigo com resultados promissores para a utilização da ELL-PBT para determinação de carbofurano em caldo de cana, o método é prático, número de etapas é menor se comparado às outras técnicas, alta frequência analítica e principalmente com baixo consumo de solventes orgânicos e de amostras (PACHECO, et al., 2017; RODRIGUES, et al., 2017). Sendo assim, essa pesquisa teve como objetivo avaliar o emprego do método (ELL-PBT) em relação à seletividade, aplicabilidade e efeito da adição de $\mathrm{NaCl}$ (cloreto de sódio) para determinação do agrotóxico carbofurano em caldo de cana e posterior análise de amostras reais de caldo de cana comercializados na cidade de Itumbiara - GO.

\section{Material e Métodos}

Todo o ensaio experimental consistiu na utilização da técnica Extração Líquido-Líquido com Partição à Baixa Temperatura (ELL-PBT), seguida de análise por CLAE-UV conforme metodologia proposta por Goulart et al. (2012). 


\section{Coleta das Amostras}

As amostras de caldo de cana utilizadas para verificação da eficiência da ELL-PBT foram obtidas através de cana-de-açúcar sem aplicação de agrotóxicos (Branco) na zona rural do município de Itumbiara - GO, Brasil. Após coleta, as amostras de cana-de-açúcar foram refrigeradas e analisadas no Laboratório de Análise Química Instrumental do Instituto Federal de Goiás Campus Itumbiara - GO, onde foram preparadas.

\section{Reagentes e Soluções}

A solução padrão estoque na concentração de 100,0 mg L ${ }^{-1}$ de carbofurano $(99,9 \% \mathrm{~m} / \mathrm{m}$ - Sigma Aldrich) foi preparada em acetonitrila (J.T. Baker, grau CLAE) e armazenada em freezer.

\section{Fortificação da Amostra e Extração do Carbofurano}

Para o processo de fortificação, adicionou-se 2,00 $\mathrm{mL}$ de caldo de cana isento de carbofurano em um frasco de vidro transparente com tampa e septo de Teflon ${ }^{\circledR}$ com capacidade de $22 \mathrm{~mL}$. Em seguida, a amostra foi fortificada com $200,0 \mu \mathrm{L}$ de solução padrão de carbofurano a $100,0 \mathrm{mg} \mathrm{L}^{-1}$, obtendo-se no extrato final 5,0 $\mathrm{mg} \mathrm{L}^{-1}$. Posteriormente, foram adicionados 4,0 $\mathrm{mL}$ de acetonitrila $(\mathrm{ACN})$, como solvente extrator. A mistura foi submetida a agitação por $60 \mathrm{~s}$ em vórtex e deixado em freezer à temperatura de $20^{\circ} \mathrm{C}$ por cerca de 3 horas para o congelamento da fase aquosa. Na Figura 2, está disposto um esquema com os procedimentos da ELL-PBT.

Em seguida, coletou-se 1,0 mL do sobrenadante e transferiu-se para vial de injeção e realizada a análise cromatográfica. A recuperação do carbofurano após fortificação do caldo de cana $5,0 \mathrm{mg} \mathrm{L}^{-1}$ foi calculada pela curva analítica construída em acetonitrila. As concentrações do carbofurano na curva foram $0,1 \mathrm{mg} \mathrm{L}$ 1, 0,5 $\mathrm{mg} \mathrm{L}^{-1}, 1,0 \mathrm{mg} \mathrm{L}^{-1}, 2,0 \mathrm{mg} \mathrm{L}^{-1}, 3,0 \mathrm{mg} \mathrm{L}^{-1}, 4,0 \mathrm{mg}$ $\mathrm{L}^{-1}$ e $5 \mathrm{mg} \mathrm{L}^{-1}$.

\begin{tabular}{|c|c|c|c|c|c|}
\hline $\begin{array}{l}2,0 \mathrm{~mL} \text { de } \\
\text { Amostras }\end{array}$ & $\begin{array}{l}\text { Adiçãã do } \\
\text { Padrão de } \\
\text { Carbofurano }\end{array}$ & $\begin{array}{c}4,0 \mathrm{~mL} \\
\text { Acetonitrila }\end{array}$ & $\begin{array}{l}\text { Agitação por } \\
60 \text { s Vórtex }\end{array}$ & $\begin{array}{c}-20^{\circ} \mathrm{C} \text { por } 3 \\
\text { horas }\end{array}$ & CLAE-UV \\
\hline
\end{tabular}

Figura 2 - Procedimentos da ELL-PBT

\section{Avaliação da Força Iônica}

Para verificar o efeito da força iônica realizou as análises em triplicata seguindo o procedimento: em frasco de vidro transparente com tampa e septo de Teflon ${ }^{\circledR}$ de $22 \mathrm{~mL}$ de capacidade, foram adicionados
2,00 $\mathrm{mL}$ de caldo de cana (isentas de carbofurano) fortificadas com padrão de carbofurano a $5,0 \mathrm{mg} \mathrm{L}^{-1}$. O estudo univariado da força iônica foi realizado com 3 repetições utilizando $1,5 \% \mathrm{~m} / \mathrm{v}$ de $\mathrm{NaCl}$ e 3 repetições sem a utilização do $\mathrm{NaCl}$. Adicionou-se $4,0 \mathrm{~mL}$ de acetonitrila, como solvente extrator. A mistura foi submetida a uma agitação durante $60 \mathrm{~s}$ em vórtex e deixado em freezer à temperatura de $-20^{\circ} \mathrm{C}$ por cerca de 3 horas para o congelamento da fase aquosa. Posteriormente, foi coletado $1,0 \mathrm{~mL}$ do sobrenadante e transferido para vial de injeção e realizada a análise cromatográfica.

Foi aplicado o teste $t$ de student (95\% de confiança) para avaliar as médias de recuperação com e sem adição de $\mathrm{NaCl}$.

\section{Condições Cromatográficas}

Foi utilizado um Cromatógrafo líquido da Marca Shimadzu, modelo LC 20AT equipado com um detector UV. As condições cromatográficas utilizadas foram: varredura espectral de $195 \mathrm{~nm}$ a $213 \mathrm{~nm}$; coluna kinetex $5 \mu \mathrm{m}$ EVO C18 - 150 x 4,6 mm; fase móvel (modo isocrático); Acetonitrila: água deionizada $35: 65 \mathrm{v} / \mathrm{v}$; vazão da fase móvel: $0,8 \mathrm{~mL} \min ^{-1}$; temperatura da coluna: $35^{\circ} \mathrm{C}$; volume de injeção: $20 \mu \mathrm{L}$; e tempo de análise: 7 minutos.

\section{Aplicação do Método em Amostras de caldo de cana no comércio}

Após a avaliação da seletividade e a aplicabilidade do método, este foi aplicado em 3 (três) amostras de caldo de cana coletados em três estabelecimentos comerciais da cidade de Itumbiara - GO, Brasil. As amostras foram analisadas em triplicatas.

\section{Resultados e Discussão}

\section{Curva Analítica}

Foram realizadas injeções de soluções padrão de carbofurano no extrato do branco da matriz do caldo de cana, com o objetivo de verificar a resposta do detector frente às diferentes concentrações do analito $\left(0,1 \mathrm{mg} \mathrm{L}^{-}\right.$ ${ }^{1}$ a $\left.5,0 \mathrm{mg} \mathrm{L}^{-1}\right)$. A partir dos dados obtidos foi construída a curva analítica $\mathbf{y}=\mathbf{2 5 1 3 1 3 x} \mathbf{- 3 2 6 2 , 7}$ com coeficiente de correlação igual a 0,9988 indicando a linearidade do detector na faixa de concentração estudada.

Os parâmetros estabelecidos pela ANVISA em sua Resolução n. 166/2017, define que o coeficiente de correlação deve estar acima de 0,990. Desta forma, nota-se que o coeficiente obtido na equação da reta deste estudo pode ser considerado um valor significativo, indicando a linearidade da resposta do detector (BRASIL, 2003; BRASIL, 2017). 


\section{Avaliação da Seletividade do Método ELL-PBT}

Na Figura 3, é apresentado a comparação do cromatograma de um extrato obtido a partir de uma amostra de caldo-de-cana (isenta de agrotóxicos) e uma amostra fortificada com solução padrão de carbofurano à $5,0 \mathrm{mg} \mathrm{L}^{-1}$. Verificou-se que o método foi seletivo, pois o cromatograma do extrato obtido após a ELL-PBT da mesma amostra sem fortificação (branco) não apresentou interferentes no mesmo tempo de retenção do carbofurano. Nota-se que os cromatogramas não apresentaram interferentes de espécies como outro ingrediente ativo, excipientes, impurezas e produtos de degradação, bem como outros compostos de propriedades similares que poderiam estar, porventura, presentes (RIBANI et al., 2004).
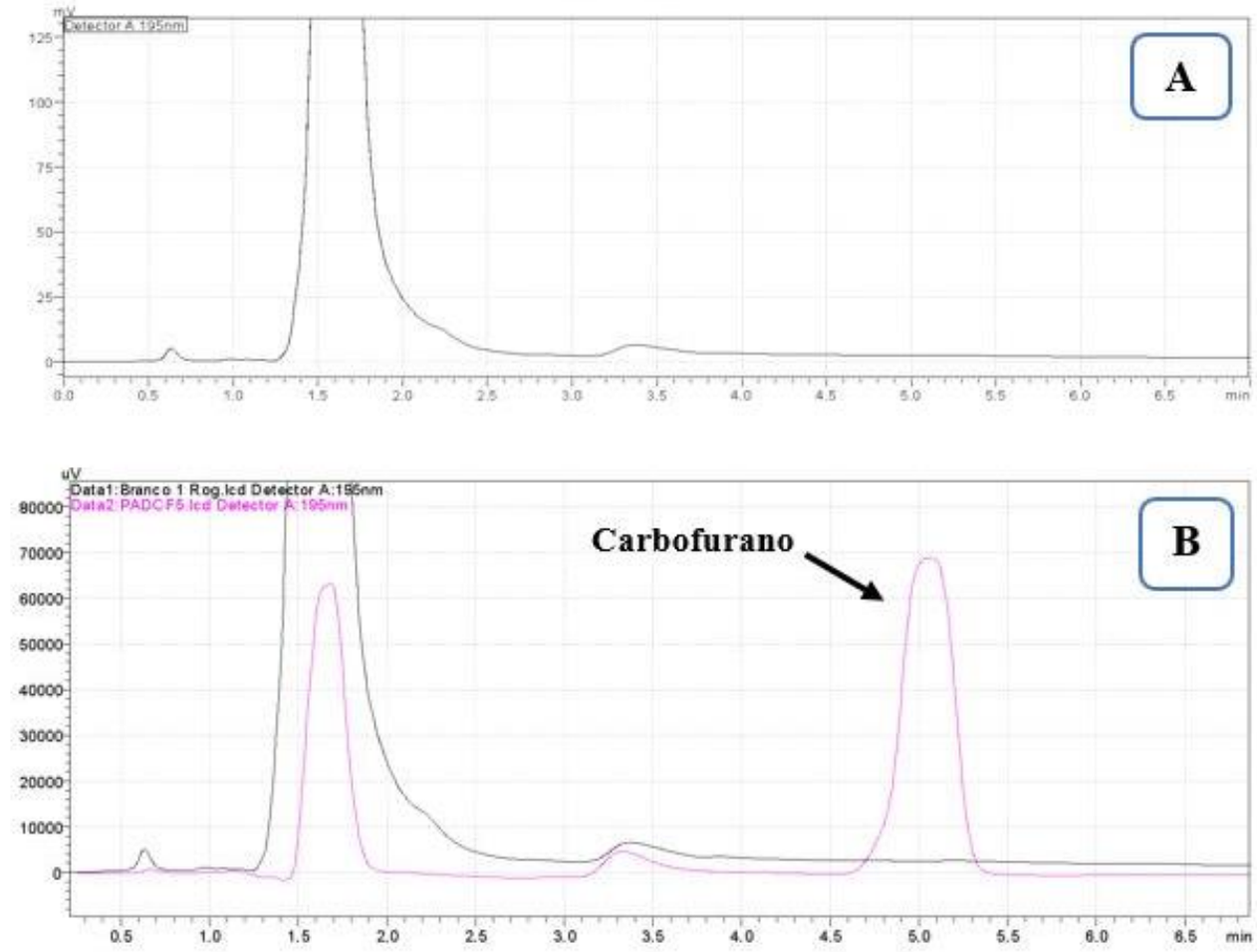

Figura 3 - (A) Cromatograma de um extrato obtido de caldo de cana isento de carbofurano e (B) Cromatograma de extrato de caldo de cana contendo carbofurano a 5,0 $\mathrm{mg} \mathrm{L}^{-1}$.

\section{Aplicação da Técnica e Avaliação da Força Iônica}

Avaliou-se a aplicabilidade do método ELL-PBT em caldo de cana através da porcentagem de recuperação do carbofurano. As porcentagens de recuperação (\%R) obtidas e os Coeficientes de Variação (CV) estão apresentados na Tabela 1 . Foi alcançada uma porcentagem média de recuperação aceitável ficando dentro da faixa de 70 a $120 \%$, conforme sugerido pelo "European Commission" no guia SANTE/11945/2017 (EUROPEAN COMMISSION, 2017). O coeficiente de variação foi de $1,87 \%$ para as amostras com adição de $\mathrm{NaCl}$ e 1,90\% para amostras sem $\mathrm{NaCl}$. Esses valores obtidos são bem menores que os aceitáveis para amostras complexas que podem ser até 20\% (ALVES, 2010). Não houve diferença significativa entre presença e ausência de $\mathrm{NaCl}$ ao nível de $95 \%$ de confiança pelo teste $t$ de student.
Tabela 1 - Porcentagem de recuperação do carbofurano

\begin{tabular}{lcccc}
\hline Ensaios & Replicatas & $\boldsymbol{\%}(\mathbf{R})$ & $\begin{array}{c}\text { Média } \\
(\boldsymbol{\%})\end{array}$ & CV (\%) \\
\hline \multirow{2}{*}{ Adição de $\mathrm{NaCl}$} & I & $101,7 \%$ & & \\
& II & $96,9 \%$ & $99,1 \%$ & $1,87 \%$ \\
\multirow{2}{*}{ Sem Adição de $\mathrm{NaCl}$} & III & $98,8 \%$ & & \\
& I & $101,5 \%$ & & \\
& III & $102,3 \%$ & $102,1 \%$ & $1,90 \%$ \\
\hline
\end{tabular}

Na pesquisa realizada por Alves (2010), utilizando a ELL-PBT na determinação de aldicarbe e carbofurano em café bebida a adição de sal também não aumentou significativamente as porcentagens de recuperação dos agrotóxicos. A adição de cloreto de sódio aumenta a força iônica da solução aquosa, facilitando a extração, devido à solvatação dos íons pelas moléculas de água, facilitando a migração dos agrotóxicos para fase 
orgânica. Porém, dependendo da matriz, a adição de $\mathrm{NaCl}$ dificulta a formação de uma única fase diminuindo a porcentagem de recuperação dos agrotóxicos (GOULART, 2010). Nesse trabalho, como não houve diferença significativa no uso do cloreto de sódio, optou-se por não utilizar na etapa de aplicação, o que reduz o uso de reagentes e também é uma etapa a menos no processo de extração.

Furlani et al. (2010) validaram o método QuEChERS para extração de sete agrotóxicos em caldo de cana. Como resultado, as análises apresentaram uma média de recuperação entre 62,9 a $107,5 \%$ e o coeficiente de variação ficou entre 5,1 e $17,6 \%$. Sendo assim, o método ELL-PBT apresentou uma melhor porcentagem de recuperação, menor consumo de solventes e uma menor variação se comparado com o método QuEChERS, desta forma, a ELL-PBT é um método promissor na extração de carbofurano em caldo de cana.

$\mathrm{Na}$ literatura, inexistem pesquisas voltadas para extração de agrotóxicos em caldo de cana utilizando ELL-PBT. Silva et al. (2017) otimizaram uma técnica semelhante para a extração de carbofurano em matrizes de cana-de-açúcar chamada Extração Sólido-Líquido com Partição a Baixa Temperatura (ESL-PBT) como resultados os pesquisadores obtiveram uma boa porcentagem de recuperação (73\%) do agrotóxico carbofurano. A mesma técnica (ESL-PBT) foi utilizada por Rodrigues et al. (2018) para extração do carbofurano em abobrinha e abacaxi (NUNES et al., 2019).

\section{Aplicação do Método de ELL/PBT e Análise por \\ CLAE/UV em Amostras de Caldo de Cana Comerciais}

As amostras de caldo de cana comercializadas no município de Itumbiara - GO, coletadas e processadas conforme descrito anteriormente foram submetidas, em triplicata, ao método otimizado de ELL-PBT e analisadas por CLAE-UV. Não foi evidenciada a presença de carbofurano nas condições instrumentais utilizadas em todas as três amostras avaliadas. O cromatograma das análises em triplicata, da amostra de caldo de cana 1 é apresentado na Figura 4. Os cromatogramas das amostras 2 e 3 foram semelhantes ao cromatograma da amostra 1.

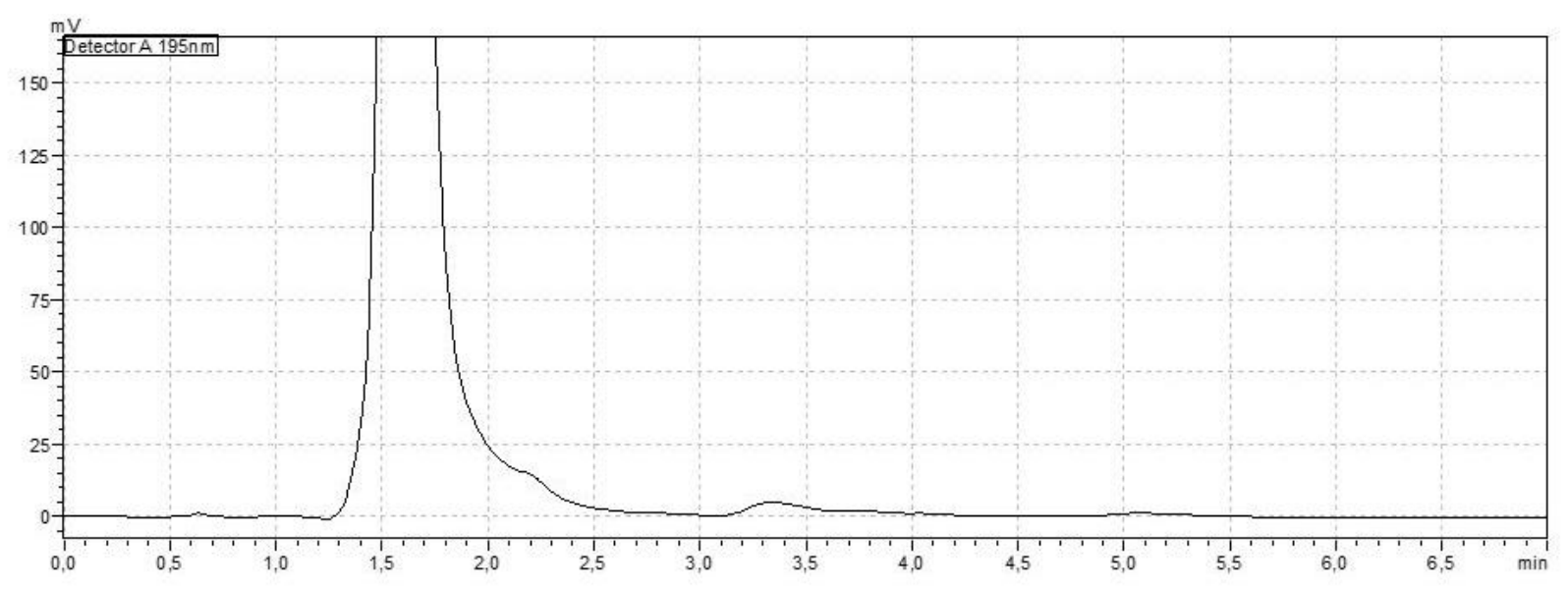

Figura 4 - Cromatograma de extrato obtido de matriz real de caldo de cana submetida à ELL-PBT e análise por CLAE-UV.

\section{Considerações finais}

Em virtude dos resultados apresentados nessa pesquisa, podemos inferir que a ELL-PBT é uma técnica seletiva para determinação de carbofurano em caldo de cana, com uma porcentagem de recuperação média de 99,18\% para amostras com cloreto de sódio e $102,19 \%$ para as amostras sem cloreto de sódio. Sendo assim, é possível inferir que o método não necessita de uma etapa adicional de adição de $\mathrm{NaCl}$. Todos os valores obtidos nesse trabalho estão dentro dos padrões estabelecidos pela "European Commission" no guia SANTE/11945/2017.

As principais vantagens dessa técnica são o baixo consumo de solvente $(4 \mathrm{~mL})$ se comparado ao método QUECHERS que pode chegar a consumir até dez vezes mais (GOULART, 2010), simplicidade na extração dos agrotóxicos, com número de etapas reduzido, possibilitando a utilização em análises rotineiras para avaliação da qualidade da bebida. Esse estudo se torna importante para incentivar a fiscalização da bebida comercializada em feiras livres e mercados populares, 
visto que atualmente não é realizado nenhum controle de qualidade. É importante ressaltar que estudos voltados para a determinação de outros agrotóxicos no caldo de cana são importantes, em razão da matéria prima (cana-de-açúcar) ser predominante na maioria dos estados brasileiros e devido ao uso intensivo de produtos químicos no combate de pragas nessas culturas.

As análises das amostras reais de caldo de cana comercializadas no município de Itumbiara - GO, utilizando a técnica ELL-PBT, não apresentaram resíduos do agrotóxico carbofurano nas condições analíticas utilizadas.

\section{Agradecimentos}

Os autores agradecem o apoio financeiro do PET/FNDE, ao Instituto Federal de Goiás Campus Itumbiara - GO e ao Núcleo de Pesquisa e Estudos em Química de Goiás (NUPEQUI).

\section{Referências}

ALCÂNTARA, D. B.; FERNANDES, T. S. M.; NASCIMENTO, H. O.; LOPES, A. F.; MENEZES, M. G. G.; LIMA, A. C. A.; CARVALHO, T. V.; GRINBERG, P.; MILHOME, M. A. L.; OLIVEIRA, A. H. B.; BECKER, H.; ZOCOLO, G. J.; NASCIMENTO, R. F. Diagnostic detection systems and QuEChERS methods for multiclass pesticide analyses in different types of fruits: An overview from the last decade. Food Chemistry, v. 298, p. 124958, 2019.

ANASTASSIADES, M.; LEHOTAY, S.; STAJNBAHER, D.; SCHENCK, F. J. Fast and easy multiresidue method employing acetonitrile extraction/partitioning and "dispersive solid-phase extraction" for the determination of pesticide residues in produce. Journal of AOAC International, v. 83, p. 412-431, 2003.

ALVES, R. D. Otimização e validação de metodologia para determinação de aldicarbe $\mathrm{e}$ carbofurano em amostras de café bebida. 2010. 153 f. Dissertação (Mestrado em Agroquímica). Universidade Federal de Viçosa, Viçosa, 2010.

ANHOLETI, M. S.; OSÓRIO, V. M.; VIEIRA, K. M. Determinação e validação de método analítico para determinação de clorpirifós em grãos de café e solo. Research, Society and Deselopment, v. 8, p. 1-16, 2019.

BARCI, P. E. P.; ALVES, L. S.; AVELLAR, A. A. S.; CENDON, L. R.; SANTOS, P. J.; STRINGHINI, F. M.; PRESTES, O. D.; ZANELLA, R. Modified QuEChRS Method for Multiresidue Determination of Pesticides in
Pecan Nuts by Liquid Chomatography Tanden Mass Spectrometry. Food Analytical Methods, v.13, p.793801, 2020.

BRASIL, Companhia Nacional de Abastecimento (CONAB). Acompanhamento da safra brasileira cana-de-açúcar. Disponível em: https://www.conab.gov.br/infoagro/safras/cana/boletim-da-safra-de-cana-de-acucar. Acesso em: 15 de março de 2020.

BRASIL, Ministério da Agricultura, Pecuária e Abastecimento (MAPA). Resumo de Registro de Agrotóxicos e Afins. Disponível em: http://www.agricultura.gov.br/assuntos/insumosagropecuarios/insumosagricolas/agrotoxicos/informacoes-tecnicas. Acesso em: 02 de fevereiro de 2020.

BRASIL, Agência Nacional de Vigilância Sanitária (ANVISA). Monografia do Carbofurano. Disponível em: http://portal.anvisa.gov.br/. Acesso em: 25 de fevereiro de 2020.

BRASIL, Agência Nacional de Vigilância Sanitária (ANVISA). Resolução da diretoria colegiada - RDC

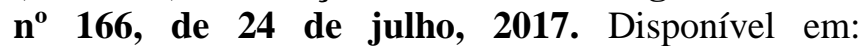
http://portal.anvisa.gov.br/documents/10181/2721567/ RDC_166_2017_COMP.pdf/d5fb92b3-6c6b-41308670-4e3263763401. Acesso em: 26 de março de 2020.

BRASIL, Instituto Nacional de Metrologia, Normalização e Qualidade Industrial (INMETRO). Orientações sobre Validação de Métodos de Ensaios Químicos, DOQ-CGCRE-008, 2003. Disponível em: http://www.inmetro.gov.br/Sidoq/Arquivos/CGCRE/D OQ/DOQ-CGCRE-8_02.pdf. Acesso em: 02 de abril de 2020 .

EUROPEAN COMMISSION. Guidance document on analytical quality control and method validation procedures for pesticide residues and analysis in food and feed. Disponível em: https://ec.europa.eu/food/sites/food/files/plant/docs/pes ticides_mrl_guidelines_wrkdoc_2017-.\%2011813.pdf. Acesso em: 15 de fevereiro de 2020.

FRAGA, W. G.; COSTA, N. R.; ALMEIDA, F. V.; REBELO, R. M.; MORAES, K. O. C.; REZENDE, J. A.; SANTANA, M. H. P.; MALDANER, A. O. Identificação dos principais ingredientes Ativos em Agrotóxicos Ilegais Apreendidos pela Polícia Federal do Brasil e Quantificação do Metsulfurom-metílico e Tebuconazol. Revista Virtual de Química, v. 8, p. 575, 2016.

FURLANI, R. P. Z.; MARCILIO, K. M.; LEME F. M.; TFOUNI, S. A. V. Analysis of pesticide residues in 
sugarcane juice using QuEChERS sample preparation and gas chromatography with electron capture detection. Food Chemistry, v. 126, p. 1283-1287, 2010.

FUMES, B. H.; ANDRADE, F. N.; SANTOS NETO, A. J.; LANÇAS, F. M. Determination of pesticides in sugarcane juice employing microextraction by packed sorbent followed by gas chromatography and mass spectrometry. Journaul of Separation Science, v. 39, p. 2823, 2016.

GALVÃO, K. N. C.; TEIXEIRA, V. M. C.; SHIMADA, L. B. C.; BAGATIN, M. C.; VALOTO, A. L. O. Análise Microbiológica do Caldo de Cana Comercializado por Vendedores Ambulantes no Município de Campo Mourão-PR. Revista de Saúde e Biologia, v.14, p. 21-26, 2019.

GOULART, S. M. Avaliação da técnica de extração com partição em baixa temperatura na análise de carbamatos em alimentos e bebidas. 2010. $136 \mathrm{f}$. Dissertação (Doutorado em Agroquímica). Universidade Federal de Viçosa, Viçosa, 2010.

GOULART, S. M; PAULAW. X; QUEIROZ, J. H; NEVES, A. A; QUEIROZ, M. E. L. R. Determination of carbamates in beverages by liquid-liquid extraction with low temperature partitioning and liquid chromatography. Journal of the Brazilian Chemical Society, v. 23, p. 1154, 2012.

GOULART, A. C.; GOULART, S. M.; MEDEIROS, M. S.; SANTOS, J. P. V. Avaliação da contaminação ambiental por carbofurano em solo proveniente do cultivo de cana-de-açúcar. TECNO-LÓGICA, V. 22, p. 187-193, 2018.

GIROUD, B.; BRUCKNER, S.; STRAUB, L.; NEUMANN, P.; WILLIAMS, G. R.; VULLIER, E. Trace-level determination of two neonicotioid insecticide residues in honey bee royal jelly using ultrasound assisted salting-out liquid liquid extraction followed by ultra-high-performance liquid chromatography-tandem mass spectrometry. Microchemical Journal, v. 151, p. 1424, 2019.

KABIR, K. H.; ABDULLAH, M.; PRODHAN, M. D. H.; AHMED, M. S.; ALAM, M. N. Determination of Carbofuran Residue in the Samples of Sugarcane (Sacharum officinarum L) and Soil of Sugarcane Field. The Agriculturists, v. 5, n. 1 e 2, p. 61-66, 2007.

LEHOTAY, S. J.; MAŠTOVSKÁ, K.; LIGHTFIELD, A. R. Use of buffering and other means to improve results of problematic pesticides in a fast and easy method for residue analysis of fruits and vegetables. Jounal of AOAC International, v. 88, p. 615, 2005.
LI, Y.; YUAN, T. Detection of pyrethroid pesticide residues in concentrate juice. Chinese Journal of Health Laboratory Technology, v18, p.1774, 2008.

LOZOWICKA, B.; IIYASOVA, G.; ZACZNSKI, P.; JANKOWSKA, M.; RUTKOWSKA, E.; HRYNKO, I.; MOJSAK, P.; SZABUNKO, J. Multi-residue methods for the determination of over four hundred pesticides in solid and liquid high sucrose content matrices by tandem mass spectrometry coupled with gas and liquid chromatograph. Talanta, v. 151, p.51-61, 2016.

MARCÍLIO, K. M.; FURLANI, R. P. Z.; TFOUNI, S. A. V. Resíduos de pesticidas sistêmicos em caldos de cana comercializados na cidade de Campinas, SP. In: Congresso Interinstitucional de Iniciação Cientifica, Campinas, Brasil, 2008.

MOTA, P. F.; MONTEIRO, L. B. O.; SHADDAY, L.; ARAÚJO, C.; MENDES, K. G. B.; REIS, A. K. V.; BRANDÃO, F. Análise parasitológica de caldo-de-cana comercializados no Distrito Federal. Revista de Divulgação Científica Sena Aires, v. 9, p. 65, 2020.

NUNES, R. C. N.; SANTOS, A. L. R.; RODRIGUES, R. P.; CARDOSO, A. T.; GOULART, A. C.; SANTOS, J. P. V.; GOULART, S. M. Avaliação da ESL-PBT na Determinação de Carbaril em Amostras de Abacaxi Pérola (Ananás Comosus) Comercializadas no Município de Itumbiara. TECNO-LÓGICA, v. 23, p. 100-107, 2019.

PACHECO RODRIGUES, R.; ALVES OLIVEIRA, Y.; VICTORINO SANTOS, J. P.; CORREIA GOULART, A.; TIMÓTEO CARDOSO, A.; CORREIA NETO, R. N.; MACHADO GOULART, S. Efeito da força iônica na análise de carbofurano em garapa utilizando o método ELL-PBT. In: Resumo $\mathbf{5 7}^{\circ}$ Congresso Brasileiro de Química, Gramado, Brasil, 2017.

PRATI, P.; CAMARGO, G. A. Característica do caldo de Cana e sua influência na estabilidade da bebida. Revisa Brasileira de Engenharia de Biossistemas, v. 2, p. 37-44, 2008.

PULGARIN, J. A.; BERMEJO, L. F.; DURÁN, A. C. Determination of carbamates in soils by liquidchromatography coupled with on-line postcolumnUV irradiation and chemiluminescence detection. Arabian Journal of Chemistry, v. 13, p. 2778-2784, 2018.

RAZA, H. A.; AMIR, R. M.; IDREES, M. A.; YASIN, M.; YAR, G.; FARAH, N.; ASSIM, M. A.; NAVEED, M. T.; YOUNUS, M. N. Residual impacto of pesticides on environment and health of sugarcane farmers in punjab with special reference to integrated pest 
management. Journal of Global Innovations in Agricultural and Social Sciences, v.7, p. 79-84, 2019.

RAMASUBRAMANIAN, T.; PARAMASIVAM, M. Persistence and metabolismo of carbofurano in the soil and sugarcane plant. Environmental Monitoring and Assessment, v.190, p. 538, 2018.

RODRIGUES, V. C; GOULART, S. M; SANTOS, J. P. V; FILHO, H. A. R; CASTRO, L. M; BERNADELI, A. A; GOMES, T. C. F. Aspectos ambientais, forenses e de saúde pública em relação ao uso de agrotóxicos em um município do estado de goiás, Brasil. Revista Sinergia, v. 17, p. 56, 2016.

RODRIGUES, A. A. Z.; QUEIROZ, M. E. L. R.; NEVES, A. A.; OLIVEIRA, A. F.; PRATES, L. H. F.; FREITAS, J. F.; HELENO, F. F.; FARONI, L. R. D. Use of ozone detergent for removal of pesticides and improving storage quality of tomato. Food Research International, v. 125, p. 108626, 2019.

RODRIGUES, R. P.; OLIVEIRA, Y. A.; NUNES R. C. N.; GOULART, S. M.; GOULART, A. C.; SANTOS, J. P. V. Avaliação do emprego do método ELL-PBT para determinação de carbofurano em caldo de cana. In: Resumo Semana de Educação, Ciência e Tecnologia de Goiás - SECITEC, Itumbiara, Brasil, 2017.

RODRIGUES, R. P.; FARIAS, W. R.; GOULART, S. M.; GOULART, A. C.; SANTOS, J. P. V.; QUEIROZ, M. E. L. R. Otimização da Extração Sólido-Líquido com Partição à Baixa Temperatura para Determinação de Carbofurano em Curcubita pepo L ("abobrinha") por Cromatografia Líquida de Alta Eficiência. Química Nova, v. 41, p. 213-2018, 2018.

RODRIGUES, A. A. Z.; NEVES, A. A.; QUEIROZ, M. E. L. R.; OLIVEIRA A. F.; PRATES, L. H. F.; MORAIS, E. H. C. Optimization and validation of the salting-out assisted liquid liquid extraction method and analysis by gas chromatography to determine pesticides in water. Eclética Química Journal, v. 43, p. 11-21, 2018.

RIBANI, M.; BOTTOCOLI, C. B. G.; COLLINS, C. H.; JARDIM, I. C. S. F.; MELO, L. F. C. Validação em Métodos Cromatográficos e Eletroforéticos. Química Nova, v. 27, p. 771-780, 2004.

SAMPAIO, M. R. F.; TOMASINI, D.; CARDOSO, L. V.; CALDAS, S. S.; DUARTE, F. A.; PRIMEL, E. G. A method for the determination of multiclass pesticides in sugarcane juice employing QuEChERS and LC-ESIMS/MS. Analytical Methods, v. 5, p. 2028, 2013.

SILVA, M. S.; CHAVES, T. A. B.; PACHECO, R. R.; GOULART, A. C.; SANTOS, J. P V.; GOULART, S.
M. Otimização da Extração Sólido-Líquido com Partição em Baixa Temperatura para Determinação de Carbofurano em Cana-de-Açúcar. Periódico Tchê Química, v. 14, p. 130, 2017.

SILVA, R. A. G. Desenvolvimento e validação de método multirresidual para determinação de agrotóxicos em mel por cromatografia líquida de ultra eficiência acoplada a espectrometria de massas em série. 2020. 132 f. Tese (Doutorado em Química). Universidade Federal de Uberlândia, Uberlândia, 2020.

SOARES, E. A.; BERNARDI, M. R. V.; BORGES, M. T. M. R. Avaliação física-química e sensorial de caldo de cana-de-açúcar (garapa) das variedades RB. Revista Brasileira de Produtos agroindustriais, v. 19, p. 291298, 2017.

SUGANTHI, A.; BHUVANESWARI, K.; RAMYA, $M$. Determination of neonicotinoid insecticide residues in sugarcane juice using LCMSMS. Food Chemistry, v. 241, p. 275-280, 2018.

VIEIRA, M. G.; STEINKE, G.; ARIAS, J. L. O.; PRIMEL, E. G.; CABRERA, L. C. C. Avaliação da Contaminação por Agrotóxicos em Mananciais de Municípios da Região Sudoeste do Paraná. Revista Virtual de Química, v. 9, p. 1800-1812, 2017.

ZHAO, L.; SZAKAS, T.; CHURLEY, M.; LUCAS, D. Multi-class multi-residue analysis of pesticides in edible oils by gas chromatography-tandem mass spectrometry using liquid-liquid extraction and enhanced matrix removal lipid cartridge cleanup. Journal of Chromatography A, v. 1584, p. 1-12, 2019. 